\title{
Casting Light on the Darkening of Colors in Historical Paintings
}

\author{
F. Da Pieve, ${ }^{1,2, *}$ C. Hogan, ${ }^{3,4}$ D. Lamoen, ${ }^{2}$ J. Verbeeck, ${ }^{2}$ F. Vanmeert, ${ }^{5}$ M. Radepont, ${ }^{5}$ \\ M. Cotte, ${ }^{6}$ K. Janssens, ${ }^{5}$ X. Gonze, ${ }^{7}$ and G. Van Tendeloo ${ }^{2}$ \\ ${ }^{1}$ ALGC, Research Group General Chemistry, Vrije Universiteit Brussel (VUB), Pleinlaan 2, B-1050 Brussels, Belgium \\ ${ }^{2}$ EMAT, University of Antwerp, Groenenborgerlaan 171, B-2020 Antwerp, Belgium \\ ${ }^{3}$ Consiglio Nazionale delle Ricerche, Istituto di Struttura della Materia (CNR-ISM), via Fosso del Cavaliere 100, 00133 Rome, Italy \\ ${ }^{4}$ Department of Physics and European Theoretical Spectroscopy Facility (ETSF), \\ Università di Roma “Tor Vergata," Via della Ricerca Scientifica 1, 00133 Rome, Italy \\ ${ }^{5}$ Department of Chemistry, University of Antwerp, Groenenborgerlaan 171, B-2020 Antwerp, Belgium \\ ${ }^{6}$ European Synchrotron Radiation Facility, Beamline ID21, BP 220, 38043 Grenoble Cedex, France \\ ${ }^{7}$ Institute of Condensed Matter and Nanoscience, Université Catholique de Louvain, Chemin des étoiles 8, bte L7.03.01, \\ B-1348 Louvain-la-Neuve, Belgium and European Theoretical Spectroscopy Facility (ETSF) \\ (Received 16 February 2013; revised manuscript received 22 August 2013; published 15 November 2013)
}

\begin{abstract}
The degradation of colors in historical paintings affects our cultural heritage in both museums and archeological sites. Despite intensive experimental studies, the origin of darkening of one of the most ancient pigments known to humankind, vermilion $(\alpha-\mathrm{HgS})$, remains unexplained. Here, by combining many-body theoretical spectroscopy and high-resolution microscopic x-ray diffraction, we clarify the composition of the damaged paint work and demonstrate possible physicochemical processes, induced by illumination and exposure to humidity and air, that cause photoactivation of the original pigment and the degradation of the secondary minerals. The results suggest a new path for the darkening process which was never considered by previous studies and prompt a critical examination of their findings.
\end{abstract}

DOI: 10.1103/PhysRevLett.111.208302

Many archeological sites, cathedral wall paintings, and famous masterpieces from major painters are affected by a slow, irreversible, light-induced degradation of pigments. Well-known examples of such phenomena are the discoloration of "chrome yellow," the pigment mostly favored by Van Gogh, and the darkening of vermilion, the red bright pigment also known as the mineral cinnabar (mercury sulfide, $\alpha-\mathrm{HgS}$ ), favored by Rubens and Italian masters and present in archeological sites worldwide. In spite of intensive experimental studies [1-7], the complex physicochemical processes underlying the degradation of vermilion remain a mystery. Factors such as light, impurities, humidity, and exposure to air are known to play a role [1], but the reasons behind their importance and the way they interconnect are not understood.

Vermilion degradation manifests through the appearance of gray or black layers, often accompanied by whitish streaks. Besides cinnabar, a number of secondary compounds have been detected in degraded samples through $\mathrm{x}$-ray absorption near-edge spectroscopy, Raman, and microscopy [2-7]: these include corderoite $\left(\alpha-\mathrm{Hg}_{3} \mathrm{~S}_{2} \mathrm{Cl}_{2}\right)$, calomel $\left(\mathrm{Hg}_{2} \mathrm{Cl}_{2}\right)$, and mercuric chloride $\left(\mathrm{HgCl}_{2}\right)$, although the presence of the latter seems to depend strongly on the sample investigated. Notably absent from these studies is the black polymorph of cinnabar, metacinnabar $(\beta-\mathrm{HgS})$, as ruled out by the most recent experimental findings [3-5]. Instead, some studies have suggested that metallic $\mathrm{Hg}$ is responsible for the dark luster [2,3,8], but its presence is highly controversial, due to the difficulty in observing crystalline elemental mercury.
PACS numbers: 82.80.Ej, 71.15.Qe, 78.20.Bh, 81.70.Fy

In this Letter, by combining ab initio theoretical spectroscopy and high-resolution microscopic x-ray diffraction, we clarify the composition of the degraded areas of the paintings and propose a thorough and coherent model that explains the microscopic origin of the combined effects of exposure to light, humidity, and air on the photoactivation and degradation of the layers. The need for a many-particle description of the excitation levels, band gaps, and optical spectra is demonstrated. While none of the compounds involved in such transformation is intrinsically gray or black, simultaneous processes of chemical origin (like photoreductive dissolution) and physical origin (structural instabilities) are activated and give rise to deposition of dark elemental $\mathrm{Hg}\left(\mathrm{Hg}^{0}\right)$ at the surface, via a path never considered before.

Experimental analysis was performed on mural samples from the Monastery of Pedralbes, one of the finest Catalan Gothic examples in Spain. Figure 1 and Table I summarize our main experimental results about the presence and in-depth distribution of the above-mentioned alteration products. The data were obtained by multimodal characterization of $\alpha-\mathrm{HgS}$ samples from a strongly degraded mural painting. Microscopic x-ray-diffraction ( $\mu$-XRD) experiments were performed at the hard-x-ray micro- and nanoprobe beam line (P06) of the PETRA III storage ring (DESY, Hamburg, Germany), using a primary energy of $18 \mathrm{keV}$. The energy was selected by means of a $\mathrm{Si}(111)$ double crystal monochromator. The beam was focused to $1.6 \mu \mathrm{m} \times 0.6 \mu \mathrm{m}$ (hor $\times$ vert) employing a KirkpatrickBaez mirror optic. Diffraction signals were recorded in 


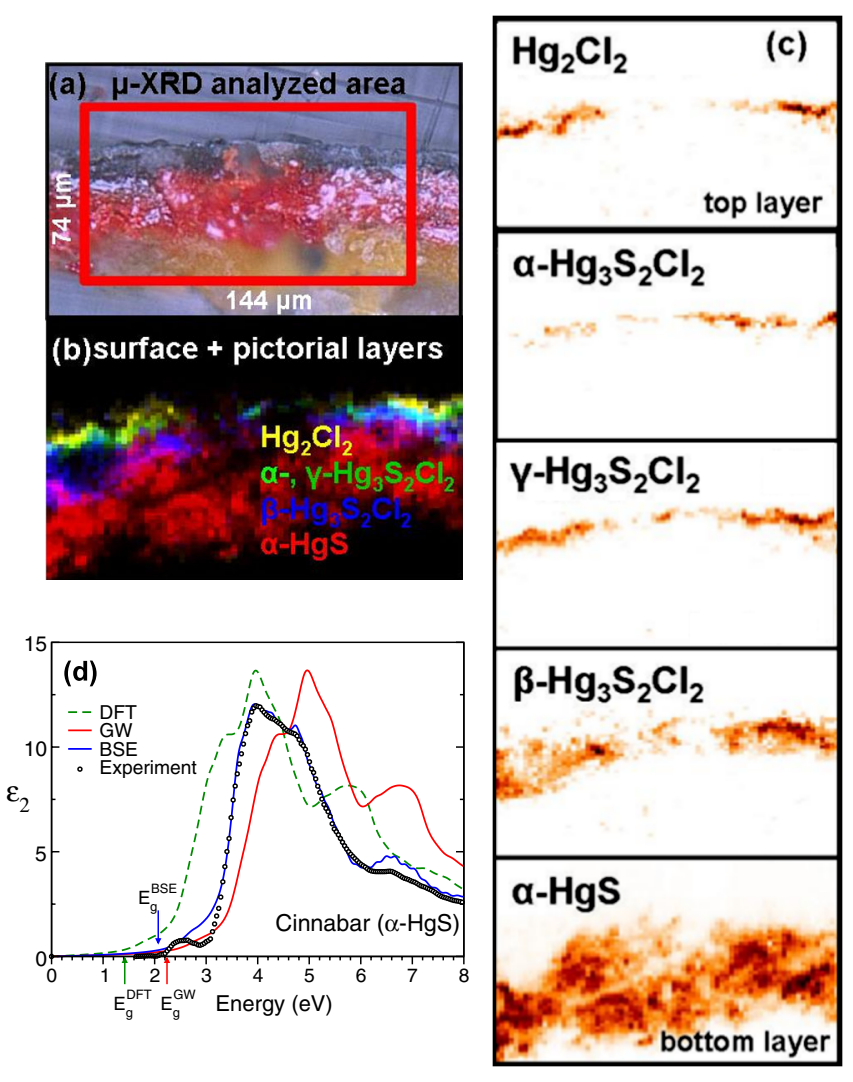

FIG. 1 (color online). (a) Degraded sample from the Monastery of Pedralbes, analyzed area as seen under ordinary light, side view; (b) high-resolution $\mu$-XRD color map, with false color, indicating the five minerals, side view; (c) $\mu$-XRD maps corresponding to Fig. 1(b), albeit separately for each of the five minerals; (d) optical absorption spectra of $\alpha-\mathrm{HgS}$ compared with experiment [33].

transmission geometry with a $2 k \times 2 k$ MarCCD area detector (MAR Research, CA, U.S.).

Figure 1(a) shows the degraded sample under investigation (side view), with the area analyzed by $\mu$-XRD indicated (the area has dimensions of $144 \times 74 \mu \mathrm{m}^{2}$, with a $2 \times 1 \mu \mathrm{m}^{2}$ step size). The red cinnabar capped by gray layers is clearly visible. The detailed sample stratigraphy, visualized by scanning $\mu$-XRD maps, is reported in Figs. 1(b) and 1(c), which show the distribution of the original red paint layer

TABLE I. Electronic band gaps (direct and indirect, where relevant) of selected compounds, in $\mathrm{eV}$.

\begin{tabular}{lccc}
\hline \hline Mineral & Cinnabar & Corderoite & Calomel \\
Formula & $\alpha-\mathrm{HgS}$ & $\alpha-\mathrm{Hg}_{3} \mathrm{~S}_{2} \mathrm{Cl}_{2}$ & $\begin{array}{c}\mathrm{Hg}_{2} \mathrm{Cl}_{2} \\
\text { White } \\
\text { Color }\end{array}$ \\
$E_{g}^{\exp }(\mathrm{eV})$ & Red & Yellow & $3.9^{\mathrm{b}}$ \\
\hline$E_{g}^{\mathrm{DFT}}(\mathrm{eV})$ & $1.40^{\text {ind }}, 1.42^{\text {dir }}$ & 1.98 & 2.06 \\
$E_{g}^{G W}(\mathrm{eV})$ & $2.24^{\text {ind }}, 2.43^{\text {dir }}$ & 2.90 & 3.52 \\
\hline \hline
\end{tabular}

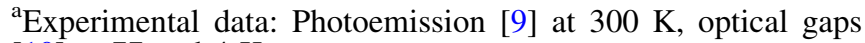
[10] at 77 and $4 \mathrm{~K}$.

${ }^{\mathrm{b}}$ Optical gap [11] at $77 \mathrm{~K}$.
$\alpha-\mathrm{HgS}$ in relation to that of several other secondary compounds, formed as a result of one or more alteration processes. Panel (b) shows a color map identifying the in-depth composition of the area analyzed, and panel (c) reports the same data as a layered stack. The XRD maps demonstrate the presence of both $\mathrm{Hg}-\mathrm{S}-\mathrm{Cl}$ and $\mathrm{Hg}-\mathrm{Cl}$ compounds localized in the degraded sample, and the coexistence of corderoite $\left(\alpha-\mathrm{Hg}_{3} \mathrm{~S}_{2} \mathrm{Cl}_{2}\right)$ with the two rare and relatively unknown phases $\gamma$ - and $\beta-\mathrm{Hg}_{3} \mathrm{~S}_{2} \mathrm{Cl}_{2}$. The detection of these polymorphs points to a critical reexamination of previous experiments and also confirms earlier suggestions about intermediate phases in the cinnabar-corderoite transformation $[12,13]$. The paint layer stack shown in Fig. 1(c) indicates that these two compounds are present close to the outer surface of the paint multilayer, in contact with the outer layer of altered material, consisting of crystalline calomel, $\mathrm{Hg}_{2} \mathrm{Cl}_{2}$ (no $\mathrm{HgCl}_{2}$ is visible in our XRD data). Nonetheless, it is important to understand from the stratigraphy that the $\beta$ phase appears mainly in the pictorial layers of the paintings, and not on the very surface, and that the formation of the sulfochloride phases occurs in the order $\beta \rightarrow \gamma$ or $\alpha$. Corderoite has been widely identified in the majority of studies as constituting the main $\mathrm{Hg}-\mathrm{S}-\mathrm{Cl}$ phase; visible effects of degradation with the passing of time (and subsequent appearance of mercury chlorides) can, thus, be studied in this polymorph.

In what follows, we present $a b$ initio calculations for cinnabar and the other main Hg-based minerals constituting the layered stack, in order to unravel (a) their original colors, (b) the microscopic mechanisms inducing the transformation from one mineral to another, and (c) the mechanisms which induce the photodarkening.

Theoretical spectroscopic results indicate that none of the minerals identified by the $\mathrm{x}$-ray analysis above is intrinsically gray or black, thus, suggesting that identification of the dark component is not possible just by looking at the crystalline composition of the degraded paintings. In Fig. 1(d), we show the optical spectrum of cinnabar computed within density functional theory (DFT), many-body perturbation theory in the $G_{0} W_{0}$ approximation [14], and through solution of the Bethe-Salpeter equation (BSE), which includes excitonic effects. All calculations were performed with the ABINIT package [15], using norm conserving pseudopotentials and the Perdew-Burke-Ernzerhof [16] parametrization of the generalized gradient approximation, and with $G_{0} W_{0}$ calculations based on the Godby-Needs plasmon-pole model. The BSE spectrum shows excellent agreement with experiment, with a relevant redistribution of the oscillator strength of the different transitions with respect to the $G_{0} W_{0}$ spectrum. However, and more importantly, the onset of the absorption occurs lower than that computed within a noninteracting quasiparticle picture $\left(G_{0} W_{0}\right)$ by less than $0.2 \mathrm{eV}$. This difference is small enough to approximate the optical gap with the electronic one, and we assume this is true for the other mercury compounds. The values for the $G_{0} W_{0}$ 
gaps for selected minerals reported in Fig. 1, together with available experimental values, indicate that such gaps are consistent with the observed red (cinnabar), yellow (corderoite), and white $\left(\mathrm{Hg}_{2} \mathrm{Cl}_{2}\right)$ color. Thus, none of these compounds being itself gray or black, the photodarkening mechanism cannot be explained on the basis of the XRD composition analysis alone, as the dark phase responsible for the degradation remains "invisible" to the corresponding XRD maps.

In order to understand quantitatively the microscopic mechanisms for the photoactivation, stratified formation, and degradation of the original and secondary minerals, we have analyzed the effect of illumination combined with exposure to a humid environment (as given by $\mathrm{Cl}$ salts, common in humid environments, salts in the air, water in the walls, and even in the protective Punic wax $[3,5]$ ) and with natural defects induced by exposure to oxygen.

Photoexcited electrons in the conduction band and partner holes in the valence band can give rise to chemical reactions (and even partial decomposition) before deexcitation, depending on the relative energy of such reactions with respect to the band edges. In order to correctly estimate the band gap values at the surface of $\alpha$ - HgS and other materials, the procedure of Ref. [17] (see also the Supplemental Material [18]) was used, i.e., DFT was employed to estimate the band edges and band gap values for the surface and these initial values were refined by employing the $G_{0} W_{0}$ results for the bulk. For the original red pigment, the valence band maximum and conduction band minimum $\left(E_{\mathrm{VBM}}\right.$, $\left.E_{\mathrm{CBM}}\right)$ for the (1010) cinnabar surface (cleavage plane [19]) with respect to the vacuum level are reported in Fig. 2. DFT calculations for the surface were performed with the quantum-ESPRESSO package [20], using norm-conserving pseudopotentials. The results for the ground state of the bulk phases were checked with those obtained by ABINIT. The surface was modeled within a supercell framework using thick $(20 \AA)$ slabs separated by vacuum.

Two key results can be obtained by comparing the band edges of the cinnabar surface with relevant redox reactions, all evaluated at $p \mathrm{H}=4$ (this value corresponds to a moderate acidic environment induced by $\mathrm{Cl}$ impurities and also

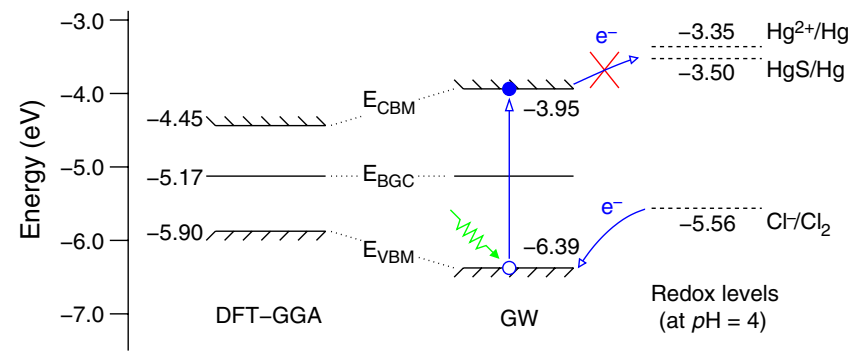

FIG. 2 (color online). Band edge positions for the $\alpha-\mathrm{HgS}(10 \overline{1} 0)$ surface (within DFT and after addition of $G W$ (i.e., $G_{0} W_{0}$ ) corrections, $\mathrm{E}_{\mathrm{BGC}}$ denotes the band gap center), and free reaction energies of $\mathrm{Cl}^{-}$oxidation and $\mathrm{Hg}(\mathrm{II})$ reduction $(p \mathrm{H}=4$, see text) corresponds to the $p \mathrm{H}$ at which the surface of cinnabar is neutral [21]). First, comparison with the potential of the reaction $\mathrm{HgS}+2 e^{-} \rightarrow \mathrm{Hg}(0)+\mathrm{S}^{2-}$ (Fig. 2) rules out the possibility that the darkening of vermilion is caused by photoinduced reduction and a possible spontaneous deposition of $\mathrm{Hg}$ at the surface. According to our result, the photoexcited electrons have, indeed, insufficient energy to directly reduce $\mathrm{Hg}^{2+}$ ions (in $\alpha-\mathrm{HgS}$ ) to $\mathrm{Hg}^{0}$. This refutes suggestions by previous experiments $[2,8]$ of an initial direct decomposition of $\alpha$-HgS itself, due to photoreductive dissolution, to give metallic $\mathrm{Hg}$ at the surface, and points to other activation mechanisms for the first step of the transformation.

The second key result is related to the role of humidity, which actually constitutes the activation mechanism under illumination. Facets exposed to light are of course also open to interaction with more harmful elements, and the presence of $\mathrm{Cl}$ in the other identified minerals yields an important clue. Our energy level diagram indicates that the cinnabar valence band edge computed with $G_{0} W_{0}$ lies well below the $\left(\mathrm{Cl}^{-} / \mathrm{Cl}_{2}\right)$ level by about $0.8 \mathrm{eV}$, allowing for oxidation of chloride impurities by photogenerated valence band holes at the cinnabar surface, and facilitating the adsorption of chlorine on the surface itself. This finding describes quantitatively the effect of humidity, as given by $\mathrm{Cl}$ salts [1]: a surface modification of the original red pigment takes place, due to quantum-allowed electron transfer mechanisms, explaining the initiation of reported transformations [1,22-24]. Although the details of the crystal growth are beyond the scope of this work, our preliminary studies show that $\mathrm{Cl}$ preferentially adsorbs on the cinnabar surface at a site consistent with its final position in the corderoite lattice [18].

We now consider the possible photoactivation mechanisms for the secondary minerals. For the sulfochlorides, we focus on corderoite. Different authors have suggested that corderoite decomposes via different paths, all involving the release of sulfur [2,3,7], which combines with atmospheric oxygen and escapes as $\mathrm{SO}_{2}$, explaining the formation of the sulfur-free phases.

Figure 3(a) shows the DFT local (relaxed) structure and density of states (DOS) around a S ion in pure corderoite, and around two structural defects likely to occur in an exposed environment: substitution of $\mathrm{S}$ by $\mathrm{O}$ and formation of $\mathrm{S}$ vacancies. For defective corderoite, we considered a $(2 \times 2 \times 2)$ supercell of the pristine material. The DOS for the $\mathrm{O}$ substitution [Fig. 3(b)] shows the presence of a defect level just below the conduction band edge. However, its shift with respect to the bottom of the conduction band is small, and more importantly, the onset of the conduction band (and, hence, the color of the material) does not change with respect to pure corderoite.

Intriguing effects come into play when considering the case of a $\mathrm{S}$ vacancy. Upon relaxation, the $\mathrm{Hg}$ vertices of the pyramidal $\mathrm{SHg}_{3}$ framework of pure corderoite form a 


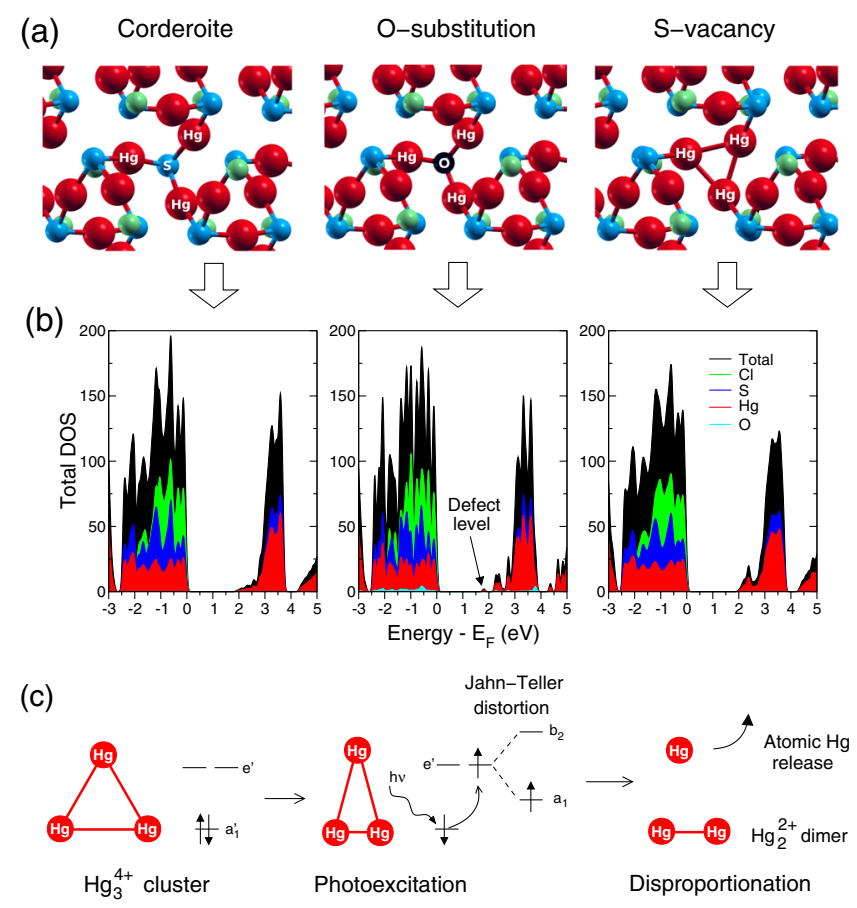

FIG. 3 (color online). (a) Atomic arrangements around a $\mathrm{S}$ atom in pure corderoite, around a $\mathrm{S}$ vacancy and around an O impurity in defective corderoite, and (b) their density of states. (c) Schematic of Jahn-Teller distortion of $\mathrm{Hg}_{3}{ }^{4+}$-like cluster [27] in defective corderoite, leading to ejection of metallic $\mathrm{Hg}$.

planar triangular $\mathrm{Hg}_{3}$ cationic cluster, in the cavity given by the vacancy [see Fig. 3(a)]. The DOS reveals the presence of a defect state ( $\mathrm{Hg} s$ type) at the conduction band edge. Analyzing the symmetry of states through crystal field and molecular orbital theory, the bonding within the $\mathrm{Hg}_{3}{ }^{4+}$ cluster in $\mathrm{D}_{3 h}$ symmetry can be considered as given by the overlap of the $s$ orbital, generating a bonding $a_{1}^{\prime}$ and two degenerate weakly antibonding orbitals $e^{\prime}$. Severe Jahn-Teller type distortions are known to occur in such $\mathrm{Hg}_{3}{ }^{4+}$ clusters when photoexcited electrons occupy such degenerate levels [25-28], leading to slow release of one $\mathrm{Hg}$ atom and formation of stable dimeric $\mathrm{Hg}_{2}{ }^{2+}$ units (see Fig. 3) [29,30]. We have, thus, analyzed within DFT the possibility of photolysis from the defective cluster structure within the supercell and found that the energy to emit one of the $\mathrm{Hg}$ atoms from the unstable fragment is $1.82 \mathrm{eV}$. This confirms that, in a molecule in solid picture, the process is well possible under both sunlight and museum lamps and could be facilitated by the large cavities in corderoite, as observed for other mercury compounds [31]. This process interconnects the three main factors of impurities, light, and exposure to air: corderoite, formed through capture of $\mathrm{Cl}$ impurities, is unstable under continuous illumination and exposure to oxygen, which act concomitantly. This structural instability constitutes the precursor factor for the formation of stable $\mathrm{Hg}_{2}{ }^{2+}$ units, the building motifs of $\mathrm{Hg}_{2} \mathrm{Cl}_{2}$, and supports our experi-

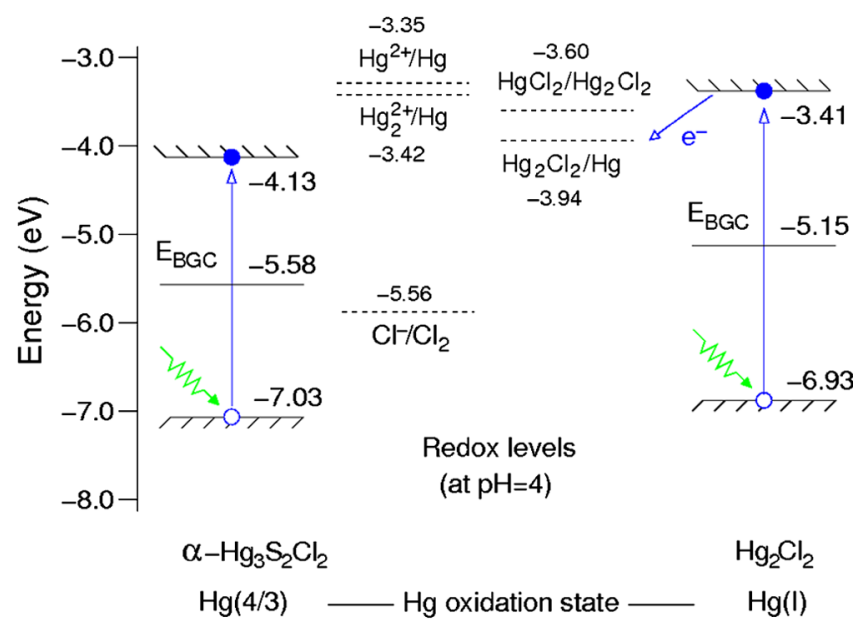

FIG. 4 (color online). $\quad G_{0} W_{0}$ electronic levels of the surface of the secondary minerals: $\alpha-\mathrm{Hg}_{3} \mathrm{~S}_{2} \mathrm{Cl}_{2}$ and $\mathrm{Hg}_{2} \mathrm{Cl}_{2}$, compared with relevant redox potentials.

mental observation of this latter phase as a natural transformation of corderoite.

A many-particle analysis of the band edges for corderoite and $\mathrm{Hg}_{2} \mathrm{Cl}_{2}$ allows us to investigate the different degradation processes occurring after the initial transformation of the original vermilion layer. $G_{0} W_{0}$ results are reported in Fig. 4, where they are compared with standard redox potentials (at $p \mathrm{H}=4$ ). For corderoite, photochemical reactions involving the reduction of its mixed valence $\mathrm{Hg}$ ions to $\mathrm{Hg}(0)$ or $\mathrm{Hg}(\mathrm{I})$ are not favored at the surface. This rules out a chemically driven path via electrontransfer mechanisms for the reported degradation of corderoite under illumination $[2,3,7,13]$ and points to structural instability as the driving factor for its degradation. However, in contrast to pure cinnabar and corderoite, we find that the electronic levels for the most likely exposed surfaces of $\mathrm{Hg}_{2} \mathrm{Cl}_{2}$ align favorably with the potential for reduction to elemental $\mathrm{Hg}$, allowing for photoreductive dissolution, i.e., decomposition via photoinduced electron transfer processes.

Thus, our results indicate that different degradation mechanisms are active in the different layers (structural instability for corderoite and electron transfer processes for calomel) and give rise to deposition of elemental $\mathrm{Hg}$ (dull gray color), which is, thus, ultimately responsible for the discoloration of the paintings. The two superimposed mechanisms cited above not only explain well the stratigraphy observed by the XRD maps, but also clarify the difficulty in identifying the origin of the photodarkening by looking at the composition of the degraded paint work, as their final by-product, $\mathrm{Hg}^{0}$, cannot be detected by conventional (room temperature) x-ray analysis, due to the unique nature of this element (a liquid metal) [32].

In conclusion, through a combination of high-resolution $\mathrm{x}$-ray diffraction and theoretical spectroscopy, we have clarified the composition of the degraded layers of the 
painting and proposed a consistent model for the pathway through which illumination, humidity, and exposure to air concomitantly affect the pigments. Our calculations elucidate the layered succession of alteration products observed by the XRD maps and indicate that the different layers are photoactivated by different mechanisms. These findings allow us to rule out previously postulated schemes involving straightforward reduction of $\mathrm{Hg}$ in cinnabar, to discriminate between different decomposition paths for corderoite, and to unravel the presence and formation of different sulfochlorides. The presence of elemental $\mathrm{Hg}$ as a by-product of the photoinduced mechanisms is proposed to cause the ultimate darkening of the paintings. Experimental verification of elemental $\mathrm{Hg}$ is, thus, strongly desired in order to provide a definitive explanation of cinnabar degradation; we suggest the application of protocols for low-temperature x-ray analysis for its detection. This work opens the way to explore the nanoscale structure and photoinduced mechanisms in various damaged artworks and to look for preservation strategies.

We gratefully acknowledge financial support from the University of Antwerp through the GOA project XANES meets ELNES (University of Antwerp Research Council), and from the SDD programme (S2-ART project) of the Belgian Federal Government (BELSPO). This work was carried out using the HPC infrastructure of the University of Antwerp (CalcUA), a division of the Flemish Supercomputer Center (VSC), which is funded by the Hercules foundation and the Flemish government (EWI department). C.H. acknowledges the CINECA Award ISCRA for the availability of HPC resources and support. J.V. acknowledges funding from the European Research Council (ERC) under the 7th Framework Program (FP7), ERC Starting Grant No. 278510 VORTEX. X. G. acknowledges support from the FRS-FNRS through the PdR Grant No. T.0238.13. G. V.T. acknowledges funding from the ERC under FP7, ERC Grant No. 246791COUNTATOMS. We express our appreciation for the help received from Dr. G. Falkenberg and his team at PETRA-III beam line P06, to DESY for granting beam time (experiments No. 10008681 and No. 10007588), and to J. Chillida and E. Pouyet for providing and preparing samples. F. D. P. and C. H. contributed equally to this work.

*Corresponding author. fabiana.dapieve@gmail.com

[1] D. Saunders and J. Kirby, National Gallery Tech. Bull. 25, 62 (2004), http://www.nationalgallery.org.uk/technicalbulletin/saunders_kirby2004.

[2] K. Keune and J. J. Boon, Anal. Chem. 77, 4742 (2005).

[3] M. Cotte, J. Susini, N. Metrich, A. Moscato, C. Gratziu, A. Bertagnini, and M. Pagano, Anal. Chem. 78, 7484 (2006).
[4] M. Cotte, J. Susini, V. Armando Sol, Y. Taniguchi, J. Chillida, E. Checroun, and P. Walter, J. Anal. At. Spectrom. 23, 820 (2008).

[5] M. Radepont, W. de Nolf, K. Janssens, G. Van der Snickt, Y. Coquinot, L. Klaassen, and M. Cotte, J. Anal. At. Spectrom. 26, 959 (2011).

[6] P. Vandenabeele, K. Lambert, S. Matthys, W. Schudel, A. Bergmans, and L. Moens, Anal. Bioanal. Chem. 383, 707 (2005).

[7] M. Spring and R. Grout, National Gallery Tech. Bull. 23, 50 (2002), http://www.nationalgallery.org.uk/technicalbulletin/spring_grout2002.

[8] B. Pal, S. Ikeda, and B. Ohtani, Inorg. Chem. 42, 1518 (2003).

[9] G. G. Roberts and R. Zallen, J. Phys. C 4, 1890 (1971).

[10] G. A. Soler, J.-P. Aicardi, and J.-P. Leyris, Phys. Status Solidi B 105, 249 (1981).

[11] S. K. Deb, Phys. Rev. B 2, 5003 (1970).

[12] E. H. Carlson, J. Cryst. Growth 1, 271 (1967).

[13] E. E. Foord, P. Berendsen, and L. O. Storey, Am. Mineral. 59, 652 (1974), http://www.minsocam.org/ammin/AM59/ AM59_652.pdf.

[14] W. G. Aulbur, L. Jonsson, and J. W. Wilkins, Solid State Phys. 54, 1 (1999).

[15] X. Gonze et al., Comput. Phys. Commun. 180, 2582 (2009).

[16] J. P. Perdew, K. Burke, and M. Ernzerhof, Phys. Rev. Lett. 77, 3865 (1996).

[17] M. C. Toroker, D. K. Kanan, N. Alidoust, L. Y. Isseroff, P. Liao, and E. A. Carter, Phys. Chem. Chem. Phys. 13, 16644 (2011).

[18] See Supplemental Material at http://link.aps.org/ supplemental/10.1103/PhysRevLett.111.208302 for technical details of the ab initio calculations.

[19] J. W. Anthony, R. A. Bideaux, K. W. Bladh, and M. C. Nichols, Handbook of Mineralogy (Mineral Data Publishing, Tucson, AZ, 2003), Vol. I.

[20] P. Giannozzi et al., J. Phys. Condens. Matter 21, 395502 (2009).

[21] M. Ravichandran, G. R. Aiken, M. M. Reddy, and J. N. Ryan, Environ. Sci. Technol. 32, 3305 (1998).

[22] J. K. McCormack, Miner. Deposita 35, 796 (2000).

[23] R.S. Davidson, C.J. Willsher, and C.L. Morrison, J. Chem. Soc., Faraday Trans. 1 78, 1011 (1982).

[24] J. K. McCormack, F. W. Dickson, and M. P. Leshendok, Am. Mineral. 76, 1715 (1991), http://www.minsocam.org/ ammin/AM76/AM76_1715.pdf.

[25] A. Vogler and H. Kunkely, Top. Curr. Chem. 213, 143 (2001).

[26] H. Zhang, Struct. Bond. 120, 37 (2006).

[27] H. Kunkely and A. Vogler, Chem. Phys. Lett. 206, 467 (1993).

[28] H. Kitamura, Chem. Phys. 325, 207 (2006).

[29] D. Rickard and G. W. Luther III, Rev. Mineral. Geochem. 61, 421 (2006).

[30] M. Kaupp and H. G. von Schnering, Inorg. Chem. 33, 4179 (1994).

[31] S. A. Magarill, N. V. Pervukhina, S. V. Borisov, and N. A. Palc̀hik, Russ. Chem. Rev. 76, 101 (2007).

[32] L. Ottaviano, A. Filipponi, and A. Di Cicco, Phys. Rev. B 49, 11749 (1994).

[33] M. Cardona, R. K. Kremer, G. Siegle, A. Muñoz, A. H. Romero, and M. Schmidt, Phys. Rev. B 82, 085210 (2010). 